Rev. Elev. Méd. vét. Pays trop., 1976, 29 (4) : 317-321.

\title{
Essai du cambendazole (*) sur des zébus nigériens âgés de 1 à 2 ans
}

\author{
par P. TAGER-KAGAN $(* *)$ \\ (avec la collaboration technique de DJIBO GARBA $\left({ }^{* *}\right)$ )
}

\begin{abstract}
RESUME
Un essai avec le Cambendazole a été pratiqué sur des zébus âgés de 1 à 2 ans au Laboratoire de Niamey.

Les résultats se sont montrés très intéressants sur les genres Haemoncus et Cooperia aux doses de $20 \mathrm{mg} / \mathrm{kg}$ et $25 \mathrm{mg} / \mathrm{kg}$; à ces doses, le médicament s'est révélé relativement efficace contre les genres Oesophagostomum et Bunostomum (le peu d'animaux parasités par ces genres d'helminthes ne permettant pas d'évaluer réellement l'efficacité du Cambendazole).

$\mathrm{L}$ a dose de $20 \mathrm{mg} / \mathrm{kg}$ a été retenue comme posologie optimale dans la lutte contre ces parasites gastro-intestinaux du zébu.
\end{abstract}

\section{INTRODUCTION}

Les helminthiases de la caillette et de l'intestin du zébu sont fréquentes au Niger ; elles sont dues à la présence dans ces organes de Nématodes, seuls ou associés appartenant aux espèces suivantes :

Haemoncus contortus,

Cooperia pectinata et Cooperia punctata, Oesophagostomum (Bosicola) radiatum,

Bunostomum phlebotomum,

Trichuris globulosa.

Pour lutter contre ces helminthiases, un grand nombre d'anthelminthiques ont été essayés avec des résultats divers; parmi eux se trouve le Cambendazole.

Le Cambendazole a pour formule brute $\mathrm{C}_{14} \mathrm{H}_{14} \mathrm{~N}_{4} \mathrm{O}_{2} \mathrm{~S}$ et pour formule développée : carbamate de isopropyl-2- (4-thiazolyl) 5-benzimidazole.

$\left.{ }^{*}\right)$ Commercialisé par Merck Sharp and Dohme.

(**) Laboratoire de l'Elevage, B. P. 485 Niamey (Rép. du Niger).
Le produit se présente sous la forme d'une poudre blanche cristalline fondant à $240^{\circ}$; très peu soluble dans l'eau mais soluble dans l'éthanol et le diméthyl-formamide. Il est parfaitement stable lorsqu'il est stocké dans des conditions normales. Le Cambendazole est rapidement métabolisé chez les bovins, la majeure partie des métabolites s'éliminant par l'urine et les fèces.

Quelques travaux ont déjà été effectués sur cet anthelminthique par HORAK, SNIJDERS et INA PIENAAR (8), EGERTON et CAMPBELL (7), RESTANI et BORRELI (9), GLANISK (10) et par DAYNES et BOUCHET (5).

Dans la perspective d'une lutte contre les Strongyloses gastro-intestinales des bovins au Niger, le Cambendazole a été testé sur des zébus nigériens âgés de 1 à 2 ans.

\section{BUT DE L'EXPÉRIMENTATION}

Il s'agissait d'évaluer l'efficacité du Cambendazole chez les zébus âgés de 1 à 2 ans contre les Nématodes gastro-intestinaux et plus spécialement contre les genres : 
- g. Haemoncus,

- g. Cooperia,

- g. Bunostomum,

- g. Oesophagostomum.

Il fallait également établir la posologie optimale.

\section{PROTOCOLE D'EXPÉRIENCE}

\section{a) Anthelminthique}

Le Cambendazole se présentait sous forme de comprimé seccable dosé à $2 \mathrm{~g}$ de produit actif par comprimé.

\section{b) Les animaux}

On a établi 4 lots de 5 animaux chacun.

Lot I : 5 animaux témoins;

Lot II : 5 animaux traités à la dose de $15 \mathrm{mg} / \mathrm{kg}$;

Lot III : 5 animaux traités à la dose de $20 \mathrm{mg} / \mathrm{kg}$;

Lot IV : 5 animaux traités à la dose de $25 \mathrm{mg} / \mathrm{kg}$.

\section{c) Méthodologie}

- Examen coproscopique et coproculture pour chaque animal avant son introduction dans les lots afin de s'assurer d'une infestation par les parasites considérés.

- Tirage au sort des animaux pour la constitution des lots.

- Coproscopie, coproculture et traitement.

- Coproscopie, coproculture et abattage des animaux 7 jours après le traitement.

Comptage des parasites.

\section{RÉSULTATS}

Les résultats ont été évalués par comparaison :

- entre le nombre d'œufs au gramme de fèces et de larves $L_{3}$ dans une goutte de suspension $(10 \mathrm{ml}$ d'eau répandue sur chaque coproculture de $5 \mathrm{~g}$ de fèces) avant le traitement et le jour de l'abattage;

— entre le nombre de parasites éliminés après le traitement et le nombre de parasites retrouvés à l'autopsie ;

- avec l'infestation des témoins.
L'efficacité du médicament a été calculée selon la formule suivante;

$100-100 \times$

Moyenne du nombre de vers trouvés

Moyenne du nombre de vers trouvés chez les traités. chez les témoins

Les résultats des examens coproscopiques et des coprocultures faits avant traitement et le jour de l'abattage sont donnés par les tableaux I et II. Le tableau III précise l'efficacité du Cambendazole selon les doses vis-à-vis des différents strongles gastro-intestinaux.

TABL. $N^{\circ} I-T a u x$ moyen $d^{\prime}$ infestation en oeufs de Strongles/gramme de fèces.

\begin{tabular}{|c|c|c|}
\hline Lot & $\begin{array}{c}\text { Examen coproscopique } \\
\text { avant traitement } \\
\text { (Méthode de stoll) }\end{array}$ & $\begin{array}{c}\text { Examen coproscopique } \\
\text { le jour d'abattage } \\
\text { (Méthode de Stoli) }\end{array}$ \\
\hline Témoin & 1180 & 880 \\
\hline $15 \mathrm{mg} / \mathrm{kg}$ & 880 & 20 \\
\hline $20 \mathrm{mg} / \mathrm{kg}$ & 580 & 0 \\
\hline $25 \mathrm{mg} / \mathrm{kg}$ & 260 & 0 \\
\hline
\end{tabular}

\section{1) Haemoncus contortus}

Aucun Haemoncus n'a été trouvé à l'autopsie des animaux traités aux doses de $25 \mathrm{mg} / \mathrm{kg}$ et $20 \mathrm{mg} / \mathrm{kg}$; la présence de larve $\mathrm{L}_{3}$ dans les coprocultures avant traitement, le rejet dans les fèces après traitement d'Haemoncus morts nous permettent d'estimer à 100 p. 100 l'efficacité du Cambendazole à ces doses.

A la dose de $15 \mathrm{mg} / \mathrm{kg}$, l'autopsie des 5 animaux traités n'a montré qu'un seul porteur d'Haemoncus (1 ver); chez les témoins il y avait un total de 3173 vers. Le pourcentage d'efficacité calculé suivant la moyenne arithmétique est égal à 99,96 p. 100 .

2) Cooperia pectinata et Cooperia punctata

Aucun parasite n'a été récolté aux autopsies des animaux traités aux doses de $15 \mathrm{mg} / \mathrm{kg}$, $20 \mathrm{mg} / \mathrm{kg}$ et $25 \mathrm{mg} / \mathrm{kg}$, malgré la présence de $\mathbf{L}_{3}$ dans les coprocultures d'avant traitement; on peut en conclure qu'à ces 3 posologies le Cambendazole est efficace à 100 p. 100 .

\section{3) Oesophagostomum (Bosicola) radiatum}

La présence de $\mathrm{L}_{3}$ dans les coprocultures avant traitement, l'élimination d'Esophagostomes morts dans les fèces après traitement, 


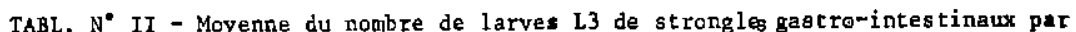
goutte de suspension des coprocultures avant le traitement et le jour de l'abattage pour chaque lat.

\begin{tabular}{|c|c|c|c|c|c|c|c|c|}
\hline \multicolumn{5}{|c|}{$\begin{array}{l}\text { Coprocultured de fèces récoltées } \\
\text { avant traitement }\end{array}$} & \multicolumn{4}{|c|}{$\begin{array}{c}\text { Coprocultures de fèces récoltées } \\
\text { le jour de l'abattage. }\end{array}$} \\
\hline & $\begin{array}{l}\mathrm{L} 3 \\
H .\end{array}$ & $\begin{array}{l}\mathrm{L} 3 \\
\mathrm{C} .\end{array}$ & $\begin{array}{l}\text { L3 } \\
B \text {. }\end{array}$ & $\begin{array}{l}\text { L3 } \\
0 .\end{array}$ & $\begin{array}{l}\mathrm{L} 3 \\
H .\end{array}$ & $\begin{array}{ll}\text { L3 } \\
\text { C. }\end{array}$ & $\begin{array}{l}\mathrm{L} 3 \\
B \text {. }\end{array}$ & $\begin{array}{l}\mathrm{L} 3 \\
0 .\end{array}$ \\
\hline $\begin{array}{l}\text { Lot traité } \\
\text { a } 15 \mathrm{mg} / \mathrm{kg}\end{array}$ & 5 & 4,4 & 0,6 & 1,2 & 0 & 0 & 0,4 & 0 \\
\hline $\begin{array}{l}\text { Lot traicé } \\
\text { a } 20 \mathrm{mg} / \mathrm{kg}\end{array}$ & 5,4 & 3,8 & 0,8 & 0,6 & 0 & 0 & 0 & 0 \\
\hline $\begin{array}{l}\text { Lot traiter } \\
\text { a } 25 \mathrm{mg} / \mathrm{kg}\end{array}$ & 3,8 & 3,8 & 0,4 & 0,2 & 0 & 0 & 0 & 0 \\
\hline Lot tëmoin & 6,4 & 5 & 0,4 & 0,4 & 6,6 & 4.4 & 0 & 0.2 \\
\hline
\end{tabular}

H. = Haemonchus; $C .=$ Cooperia; $B .=$ Bunostomum $; 0$. = Oesophagostomom.

TABL, $N^{\circ} I I I$ - Efficacité du Cambendazole.

\begin{tabular}{|c|c|c|c|}
\hline \multirow[b]{2}{*}{ Haemonchua } & \multicolumn{3}{|c|}{ Nombre de parasites récoltés à l'autopsie } \\
\hline & $\begin{array}{l}\text { Moyenne } \\
\text { arithmétique }\end{array}$ & $\begin{array}{c}\text { Nombre d'animaux } \\
\text { parasités }\end{array}$ & $\begin{array}{l}\text { Nombre total } \\
\text { de paresites }\end{array}$ \\
\hline Lot tëmoin & 634,6 & $5 / 5$ & 3173 \\
\hline $\begin{array}{l}\text { Lot traité à } 15 \mathrm{mg} / \mathrm{kg} \\
\text { Efficecitê p. } 100\end{array}$ & $\begin{array}{c}0,2 \\
99,96\end{array}$ & $1 / 5$ & 1 \\
\hline $\begin{array}{l}\text { Lot traitê à } 20 \mathrm{mg} / \mathrm{kg} \\
\text { Efficacité p. } 100\end{array}$ & $\begin{array}{r}0 \\
100\end{array}$ & $0 / 5$ & 0 \\
\hline $\begin{array}{l}\text { Lot traité à } 25 \mathrm{tgg} / \mathrm{k}_{\mathrm{B}} \\
\text { Efficacitê p. } 100\end{array}$ & $\begin{array}{r}0 \\
100\end{array}$ & $0 / 5$ & 0 \\
\hline $\begin{array}{l}\text { Cooperia } \\
\text { Lot témoin }\end{array}$ & 712,8 & $5 / 5$ & 3564 \\
\hline $\begin{array}{l}\text { Lot traitë a } 15 \mathrm{mg} / \mathrm{kg} \\
\text { Efficacité p. } 100\end{array}$ & $\begin{array}{r}0 \\
100\end{array}$ & $0 / 5$ & 0 \\
\hline $\begin{array}{l}\text { Lot traité à } 20 \mathrm{mg} / \mathrm{kg} \\
\text { Efficacitê p. } 100\end{array}$ & $\begin{array}{r}0 \\
100\end{array}$ & $0 / 5$ & 0 \\
\hline $\begin{array}{l}\text { Lot traitê à } 25 \mathrm{mg} / \mathrm{kg} \\
\text { Efficacitê p. } 100\end{array}$ & $\begin{array}{r}0 \\
100\end{array}$ & $0 / 5$ & 0 \\
\hline $\begin{array}{l}\text { Oesophagostomum } \\
\text { Lot témoin }\end{array}$ & 9,4 & $3 / 5$ & 47 \\
\hline $\begin{array}{l}\text { Lot traité à } 15 \mathrm{mg} / \mathrm{kg} \\
\text { Efficacitê p. } 100\end{array}$ & $\begin{array}{r}0 \\
100\end{array}$ & $0 / 5$ & 0 \\
\hline $\begin{array}{l}\text { Lot traité à } 20 \mathrm{mg} / \mathrm{kg} \\
\text { Efficacité p. } 100\end{array}$ & $\begin{array}{r}0 \\
100\end{array}$ & $0 / 5$ & 0 \\
\hline $\begin{array}{l}\text { Lot traité à } 25 \mathrm{mg} / \mathrm{kg} \\
\text { Efficacité p. } 100\end{array}$ & $\begin{array}{r}0 \\
100\end{array}$ & $0 / 5$ & 0 \\
\hline $\begin{array}{l}\text { Bronos tomum } \\
\text { Lot tëmoin }\end{array}$ & 17,2 & $3 / 5$ & 86 \\
\hline $\begin{array}{l}\text { Lot traitê à } 15 \mathrm{mg} / \mathrm{kg} \\
\text { Efficacitê p. } 100\end{array}$ & $\begin{array}{c}34,6 \\
0\end{array}$ & $3 / 5$ & 173 \\
\hline $\begin{array}{l}\text { Lot traité à } 20 \mathrm{mg} / \mathrm{kg} \\
\text { Efficacitê p. } 100\end{array}$ & $\begin{array}{r}1,2 \\
93,0\end{array}$ & $3 / 5$ & 6 \\
\hline $\begin{array}{l}\text { Lot traitê à } 25 \mathrm{mg} / \mathrm{kg} \\
\text { Efficacitê p. } 100\end{array}$ & $\begin{array}{r}4,2 \\
75,5\end{array}$ & $1 / 5$ & 21 \\
\hline
\end{tabular}


l'absence de parasite à l'autopsie des animaux traités tendraient à prouver qu'aux doses de $15 \mathrm{mg} / \mathrm{kg}, 20 \mathrm{mg} / \mathrm{kg}, 25 \mathrm{mg} / \mathrm{kg}$ le Cambendazole est efficace à 100 p. 100 sur les CEsophagostomes adultes. Par contre, le médicament semble inactif sur les laryes intranodulaires d'Oesophagostomum; en effet, il a été retrouvé des larves $\mathbf{L}_{4}$ vivantes à l'ouverture des nodules asophagostomiens sur le cocum des animaux témoins et des animaux traités aux différentes doses.

\section{4) Bunostomum phlebotomum}

A la dose de $15 \mathrm{mg} / \mathrm{kg}$, le Cambendazole est inefficace contre ce parasite.

A la dose de $20 \mathrm{mg} / \mathrm{kg}$, le pourcentage d'efficacité suivant la moyenne arithmétique est de 93 p. 100 (77 p. 100 en utilisant la moyenne géométrique). A la dose de $25 \mathrm{mg} / \mathrm{kg}$, le pourcentage d'efficacité selon les moyennes arithmétique et géométrique est de 75,5 p. 100 et 70,7 p. 100.

5) Comme les autres anthelminthiques dérivés du groupe benzimidazole, le Cambendazole a une action ovicide sur les ceufs de strongles gastro-intestinaux; en effet, aucune larve $\mathrm{L}_{3}$ ne s'est développée dans les coprocultures de fèces d'animaux qui contenaient des œufs de strongles à l'examen coproscopique le jour suivant le traitement.

\section{INTERPRÉTATION DES RÉSULTATS}

Sur Haemoncus contortus, Cooperia pectinata et Cooperia punctata le Cambendazole aux doses de $20 \mathrm{mg} / \mathrm{kg}$ et $25 \mathrm{mg} / \mathrm{kg}$ a une efficacité de 100 p. 100.

Sur 20 animaux d'expérience, 11 seulement étaient parasités par des Esophagostomes; le nombre total d'Helminthes récoltés était de 45 vers. Bien qu'aucun parasite n'ait été trouvé à l'autopsie des animaux traités, vu le petit nombre d'animaux parasités $(55$ p. 100$)$ et le faible niveau de l'infestation, il nous est difficile de donner un pourcentage d'efficacité du Cambendazole sur les Esophagostomes adultes; nous dirons seulement que le Cambendazole est efficace aux doses de $15 \mathrm{mg} / \mathrm{kg}, 20 \mathrm{mg} / \mathrm{kg}$ et $25 \mathrm{mg} / \mathrm{kg}$ sur les parasites adultes du genre Oesophagostomum.

L'interprétation de l'efficacité du Cambendazole sur le genre Bunostomum est également délicate; en effet, le peu d'animaux parasités (50 p. 100) semble être responsable des distorsions observées dans le calcul de l'efficacité suivant la moyenne arithmétique ou la moyenne géométrique; il semble cependant que le Cambendazole aux doses de $20 \mathrm{mg} / \mathrm{kg}$ et $25 \mathrm{mg} / \mathrm{kg}$ soit modérément efficace contre les helminthes du genre Bunostomum.

\section{CONCLUSIONS}

Cet essai du Cambendazole sur des zébus nigériens âgés de 1 à 2 ans, infestés naturellement par des strongles gastro-intestinaux, a montré que le médicament était très efficace (100 p. 100) sur les genres Haemoncus et Cooperia, efficace contre les vers adultes du genre Oesophagostomum et modérément efficace sur le genre Bunostomum aux doses de $20 \mathrm{mg} / \mathrm{kg}$ et $25 \mathrm{mg} / \mathrm{kg}$.

D'un point de vue pratique, nous recommandons d'utiliser la dose de $20 \mathrm{mg} / \mathrm{kg}$ lors de traitement systématique de masse contre les strongyloses gastro-intestinales du zébu.

- Nous remercions le Docteur MEGARD (Merck Sharp and Dohme) pour l'aide qu'il nous a apportée lors de cet essai.

\section{SUMMARY}

Trial with Cambendazole in Nigerian zebu cattle (one to two years old)

A trial with Cambendazole had been carried out in zebu cattle (one to two years old) in the Laboratory of Niamey. The results are of great interest against Haemoncus and Cooperia at dosage level of $20 \mathrm{mg} / \mathrm{kg}$ and $25 \mathrm{mg} / \mathrm{kg}$ bodyweight; at these doses the drug has revealed a relative efficacy against Oesophagostomum and Bunostomum (few animals parasited by these genus had not allowed to estimate really the efficacy of Cambendazole).

$20 \mathrm{mg} / \mathrm{kg}$ bodyweight is recommended as dosage for the treatment against gastro-intestinal strongylidae of zebu cattle. 


\section{RESUMEN}

\section{Ensayo del Cambendazole sobre cebues de Niger de 1 a 2 años de edad}

Se efectuó un ensayo con el Cambendazole en cebues de 1 a 2 años de edad en el laboratorio de Niamey.

Las dosis de $20 \mathrm{mg} / \mathrm{kg}$ y $25 \mathrm{mg} / \mathrm{kg}$ daron resultados muy interesantes contra los generos Haemoncus y Cooperia.

El medicamento fue relativamente eficaz contra Oesophagostomum y Bunostomum (pocos animales estando infestados por dichos generos de helmintos no se puede determinar realmente la eficacia del Cambendazole).

Se aconseja la dosis óptima de $20 \mathrm{mg} / \mathrm{kg}$ en la lucha contra estos parásıtos gastro-intestinales del cebú.

\section{BIBLIOGRAPHIE}

1. BENZ (G. W.). Anthelmintic activities of Cambendazole in calves. Am. J. vet. Res., 1971, $32: 399-403$.

2. BENZ (G. W.). Activity of Cambendazole against gastro-intestinal nematodes of calves: single vstwo therapeutic treatments. $J$. Parasit, 1971, 57 (2) : 286-288.

3. CAMPBELL (W. C.), BUTTLER (R. W.). Efficacy of Cambendazole against tapeworms and rounworms in lambs. Am. Soc. Parasit. 48th Ann-Mig Univ. Toronto, 1973, June 25-29, p. 45.

4. CIORDA (H.), Mc CAMPBELL (W. C.). Anthelmintic efficacy of four doses levels of Cambendazole in cattle. Proc. Helminth. Soc., Wash., 1971, 38 : 40-42.

5. DAYNES (P.), BOUCHET (A.). Essais du Cambendazole sur des veaux malgaches. Rapport I. E. M. V. T. Tananarive, 1970.

6. EGERTON (J. R.) et collab. The efficacy of Cam- bendazole against gastro-intestinal nematodes of cattle. Res. vet. Sci., 1970, $11: 495-499$.

7. EGERTON (J. R.), CAMPBELL (W. C.). The efficacy of 5 isopropoxy-carbonylamino-2-4 (Thizolyl) benzimidazole against helminths of Sheep. Res. vet. Sci., 1970, 11 : 193-195.

8. HORAK (I. G.), SNIJDERS (A. J.), PIENAAR (I.). The efficacy of Cambendazole against cestode and nematode infestation in Sheep and cattle. $J . S$. Afr. vet. Ass., 1972, 43 (1) : 101-106,

9. RESTANI (R.), BORRELI (D.). Investigations of the activity of Cambendazole in natural Strongyloides papillosus infection in calves. Vet. Ital., 1971, 22 : 145-147.

10. SIBALIC (S.), LEPOJEV (O.), MIKLIJAN. Effet du Cambendazole sur Dicrocoelium dentriticum chez le mouton naturellement infesté. Veterinarski Glasnik., 1971, 25 (11) : 835-839. 